\title{
Clinical Practicability of Extending the Length of Gastrointestinal Decompression Cathetering
}

\author{
Lei SUN ${ }^{1}$, Xin-Quan GU ${ }^{1,}$ a , An-Na WANG ${ }^{2}$, Fan-Ping MENG ${ }^{1, b, ~ * ~}$ \\ ${ }^{1}$ Department of Urology,China-Japan Union Hospital,Jilin University, Changchun,Jilin, China \\ ${ }^{2}$ Department of Hepatology, Jilin Academy of Traditional Chinese Medicine, Changchun, Jilin, \\ China \\ aemail:guxinq1213@sohu.com, bemail:947410581@qq.com \\ ${ }^{*}$ Corresponding author
}

Keywords: Gastrointestinal decompression, Stomach tube.

\begin{abstract}
Objective: We intended to investigate the appropriate length of the stomach tube in the treatment of gastrointestinal decompression. Material and Methods: The patients in treatment of gastrointestinal decompression cured by our department from January 2013 to May 2015 whom were divided into 2 groups randomly. Results: The average remission rate was $52.4 \%$ vs $14.3 \%(\mathrm{P}<0.05)$. Conclusions: The length of the catheterization largely effect the abdominal distention remission rate, which is should be $55-65 \mathrm{~cm}$ with $10 \mathrm{~cm}$ longer than the traditional.
\end{abstract}

\section{Introduction}

Gastrointestinal decompression uses the negative pressure attraction principle, and stomach tube is inserted from the oral cavity or nasal cavity. The gas or liquid accumulated in the gastrointestinal tract through the stomach tube will be sucked, relieving the pressure in the gastrointestinal tract. Effective gastrointestinal decompression can reduce gastric pressure, reduce abdominal distention, lighten the thread tension and incision pain, improve the intestinal wall blood circulation, and promote digest function recovery. It is the nursing technical operation in the application of clinic. According to the requirements of the textbooks, catheterization length of adults is about $45-55 \mathrm{~cm}$, but when the nurses indwell catheter for patients clinically, gastric juice often can't be drained out or drainage is inadequate. Postoperative patients appear different degrees of abdominal distention, affecting the postoperative recovery. The patients in treatment of gastrointestinal decompression cured by our department from January 2013 to May 2015 were randomly divided into two groups, reported as follows. 


\section{Material and Methods}

\section{Clinical Data}

42 cases of patients (conscious adults) in treatment of gastrointestinal decompression cured by our department from January 2013 to May 2015 were randomly divided into the experimental group and the control group $(n=21)$ (there is no difference in gender, age, height, bodily form, basic diseases in two groups).

\section{Methods}

Traditional catheterization length of adults from the nasal tip to the earlobe to xiphoid is about $45-55 \mathrm{~cm}$ in the control group. The catheterization length from the nasal tip to the earlobe to xiphoid adding the length from the nasal tip to the bun is about $55 \sim 65 \mathrm{~cm}$ in experimental group. Observe abdominal distension and drainage situation of two groups of patients within $24 \mathrm{~h}$ after the gastric intubation.

\section{Abdominal Distension Standard}

None: abdominal flat, soft with no tenderness; abdominal respiration is normal. Mild: stomach flat with a little tenderness; there is no abdominal respiration. Moderate: abdominal distention slightly with slight tenderness; abdominal respiration weakens. Severe: the abdomen of obvious, abdominal tenderness, no rebound tenderness; abdominal respiration weakens obviously or disappears.

\section{Result}

The four-fold table chi-square test after numerical correction finds that compare the experimental group with control group, the former can obviously improve the volume of drainage and remit the abdominal distension within $24 \mathrm{~h}$. There is statistical significance $(\mathrm{P}<$ 0.05).Table 1 shows the abdominal distension remission rate and table 2 shows the volume of drainage.

Table 1.

\begin{tabular}{|c|c|c|}
\hline \multirow{2}{*}{ Parameters } & \multicolumn{2}{|c|}{ abdominal distension } \\
\hline & existence & remission \\
\hline $\begin{array}{c}\text { Experimental } \\
\text { group }(55-65 \mathrm{~cm})\end{array}$ & 10 & 11 \\
\hline Control group $(45-55 \mathrm{~cm})$ & 18 & 3 \\
\hline $\mathrm{P}$ & & \\
\hline
\end{tabular}

Compared with Control group: ${ }^{\mathrm{P}}<0.05$, ** $\mathrm{P}<0.01$ 
Table 2.

\begin{tabular}{ccc}
\hline Parameters & \multicolumn{2}{c}{ Volume of drainage for 24 hours } \\
& $>400 \mathrm{ml}$ & $<400 \mathrm{ml}$ \\
\hline $\begin{array}{c}\text { Experimental } \\
\text { group(55-65cm) } \\
\text { Control group }(45-55 \mathrm{~cm}) \\
\mathrm{P}\end{array}$ & 18 & 3 \\
\hline
\end{tabular}

Compared with Control group:* $\mathrm{P}<0.05, * * \mathrm{P}<0.01$

\section{Discussion}

Human anatomy shows the average esophageal length is $25-30 \mathrm{~cm}$, pharyngeal length is 12 $\mathrm{cm}$, nasal length is about $8 \mathrm{~cm}$, with total length of $45-50 \mathrm{~cm}$. The distance of distal gastric tube side hole to the top is $12.6 \mathrm{~cm}$, and the traditional catheterization length is $45-55 \mathrm{~cm}$. So the holes on stomach tube can't be completely indwelled into the stomach. This conclusion was confirmed during intraoperative indwelling gastric tube, observed that the catheterization depth just reached to gastric cardia and gastric body in control group. In the experimental group the top of the stomach tube reached the gastric antrum, and the holes were all in the stomach, while the stomach tube were not folded and twisted in the stomach. After surgery, patients with stable blood pressure adopt half supine position, and the stomach fluid was accumulated in the gastric antrum. If the catheterization depth was not enough, the distal stomach tube would only reach the bottom of the gastric body or stomach, and part of the side openings were located in the stomach esophagus cardia above, influencing liquid and gas drainage in the stomach, which can't play a good effect of gastrointestinal decompression, is easily to cause stomach expansion, increase the anastomotic tension, and affect the healing of sutures.

Dai Haiping observed under ultrasound, the catheterization length was $45 \sim 55 \mathrm{~cm}$, the top of the stomach tube only reached the bottom of the gastric body or stomach, part of the side openings were within the esophagus, and gastric contents cannot be fully contacted, not reaching the ideal effect of gastrointestinal decompression [1]. Yang Huiyin observed under gastroscope, found the same result [2]. Yi Xianmei found that if the catheterization length was $55-65 \mathrm{~cm}$, then it can make the stomach tube side opening enter into the stomach to gastric antrum, making large range of forming negative pressure and low pressure in the stomach, enternal gas and liquid can flow into the stomach to be drained [3].

Literature reported, the depth of gastrointestinal decompression tube with all tube side openings in the stomach was 55 65 $\mathrm{cm}$, which also proved that tube side openings can completely reach the stomach, with a good decompression effect, and the catheterization length must be at least $55 \mathrm{~cm}$. Reviewed the past, after inserting the gastric tube, only a small amount of gastric juice can be took out, sometimes only a small amount of mucus without gastric juice was pulled out, and gurgling existed in the stomach when auscultation. Although stomach tube is proved to be in the stomach, postoperative decompression effect was not good, patients had abdominal distention and gastric peristalsis reverted slowly. 
From January 2013 to December 2014, we adopted catheterization length of $55 \sim 65 \mathrm{~cm}$ on all patients in treatment of gastrointestinal decompression, after the treatment with the method gastrointestinal decompression through the method, patients' symptoms such as abdominal distension, abdominal pain significantly reduced. It should be popularized clinically.

\section{Conclusion}

The length of the catheterization largely effect the abdominal distention remission rate, which is should be $55 \sim 65 \mathrm{~cm}$ with $10 \mathrm{~cm}$ longer than the traditional.

\section{References}

[1] Dai Haiping. Research Progress of Adult Stomach Tube Insertion Depth [J]. Modern Nurses, 2011, 8:7

[2] Yang Huiyin, Du Lenuan, Liu Chunxia. Discussion of gastric canal insertion length during adult stomach tube decompression [J]. Jilin Medical Journal, 2006, 27(4): 395

[3] Yi Xianmei, Xue Hongda. Discussion of gastric canal insertion length during stomach tube decompression [J]. National Medical Frontiers of China, 2009,4(21): 79 